\title{
Analytical challenges in nanomedicine
}

\author{
Christine Kranz • Douglas C. Eaton • Boris Mizaikoff
}

Published online: 12 January 2011

(C) Springer-Verlag 2011

Nanomedicine is a rapidly developing interdisciplinary research area bridging biology, medicine, engineering, chemistry, material science, and nanotechnology. In recent years, nanotechnology has developed an increasing impact on medical research and practice by taking advantage of the unique properties and dimensions of nanoscale materials, structures, and devices. New approaches applying nanotechnology in medicine are continuously being discovered and range from the localized application of therapeutics via nanoparticles to single molecule detection at the cellular level. Hence, the emerging research area of nanomedicine focuses on nanoscale science and technology facilitating the diagnosis, treatment, and prevention of disease and traumatic injury, the relief of pain, and the preservation and improvement of human health utilizing molecular level tools providing advanced insight into the associated biological processes.

Consequently, taking advantage of the nanotechnology revolution in the medical sciences requires the develop-

Published in the special issue Analytical Challenges in Nanomedicine with Guest Editors Boris Mizaikoff, Douglas C. Eaton, and Christine Kranz.

C. Kranz $\cdot$ B. Mizaikoff $(\bowtie)$

Institute of Analytical and Bioanalytical Chemistry, University of Ulm,

Albert-Einstein-Allee 11,

89081 Ulm, Germany

e-mail: boris.mizaikoff@uni-ulm.de

C. Kranz

e-mail: christine.kranz@uni-ulm.de

D. C. Eaton

Department of Physiology, Emory University School of Medicine, 615 Michael Street, Suite 648,

Atlanta, GA 30322-3110, USA

D. C. Eaton

e-mail: deaton@emory.edu ment of complementary analytical techniques and methods for probing health relevant biological processes, disease pathologies, and therapeutic progress at a cellular and molecular scale with appropriate spatial and temporal resolution. The importance of this field was recognized by government agencies and institutions such as the Food and Drug Administration USA, which cited nanomedicine as an area of future priority in its 2006 report [1]. Furthermore, large-scale initiatives in this field are supported by the National Institutes of Health in the USA, which funds eight so-called Nanomedicine Development Centers [2] and by the European Union within a variety of initiatives. For example, within the EuroNanoMed Joint Transnational Calls in 2009 and 2010, 17 million Euros of research funding were allocated for research projects in regenerative medicine, diagnostics, and targeted delivery systems taking advantage of nanotechnology; these initiatives will be followed by a third call in 2011. The European Science Foundation has documented the potential benefits of nanomedicine in a series of reports providing a forward perspective on nanomedicine starting in 2003 [3].

However, the emerging field of nanomedicine faces several challenges including the general complexity of biological systems interacting with nanoscale materials and devices, the translation of novel measurement concepts and methods into daily clinical routine, and concerns about the safety, potential toxicity, and efficacy of nanoscale medical devices, diagnostic techniques, and materials [4]. In general, it is evident that the majority of research published under the umbrella of nanomedicine is related to nanoparticles and polymeric drug release, which are probably the topics with the most imminent impact in this field. Currently, there are more than 400 ongoing clinical trials involving nanotechnology; the majority of which are for cancer treatment [5]. 
A literature search using the keywords "nanomedicine" and "analytical chemistry" reveals that to date only a very small fraction of the published literature addresses not only the needs and challenges, but also the potential research opportunities for analytical sciences in this emerging field $[6,7]$. From the analytic chemistry point of view, we may consider individual cells as a measurement compartment with spatial/volume dimensions in the $\mu \mathrm{m}-\mathrm{nm} / \mu \mathrm{L}-\mathrm{nL}$ range and quantitative molecular dimensions in the $\mathrm{mM}-\mathrm{nM}$ domain. The localized nature of molecular events within or at cellular compartments (e.g., vesicular processes) or at the cell surface (e.g., exo- or endocytosis) along with the local concentration of the constituents of interest determines the need for quantitative analytical measurements at the submicro- and nanoscale. Yet, the diversity of measurement challenges at these compartments include the small dimensions of the involved samples and volumes, the complex and frequently changing background matrix, potential limitations in the sensitivity and/or discriminatory power of in situ analytical techniques, and their temporal and/or spatial resolution to monitor quantitatively dynamic processes associated with cellular functions. In turn, individual optical/ spectroscopic, electrochemical, and surface sensitive analytical techniques have already demonstrated significant potential at the micro- to nanoscopic level enabling the identification of molecular species that are present, their concentration, their location, and-ideally-the kinetics/ dynamics of the involved molecular processes.

Beyond the technological barriers, a significant challenge in translating nanoscale methods into medically relevant tools results from the disparity in scientific culture separating medical disciplines from the physical sciences. Traditionally, for example, experts in cellular signaling and signal transduction are well versed in applying biochemical methods and molecular approaches to address biological problems relevant to health sciences. However, based on their background, they may not take immediate advantage of the rapid evolution in chemical, physical, and material sciences facilitating the usage of more sophisticated nanoscale technologies and methods for addressing complex problems within their discipline. In turn, scientists advancing nanotechnology may not be aware of the most pertinent questions and analytical challenges to be addressed in cell physiology or-more generally-in biomedicine.

During the preparation of this special issue, it became evident that it is indeed difficult to identify the current analytical challenges in nanomedicine as a collection of biomedical problems to be solved. The variety of topics addressed by the authors ranging from microelectrode arrays and optical sensing/assay techniques to quantum dots and scanning probe microscopies only provides a snapshot of the available (nano)analytical toolset for investigating cell physiology or disease-relevant pathophysiology at an appropriate temporal and spatial scale. Yet, the early detection of pathological changes at individual cells, cell ensembles or entire tissues via molecular and/or morphological patterns, the detection and tracing of key molecular markers, and the - ideally label-free - analysis of and within sub-cellular compartments remain analytical challenges demanding methods with enhanced sensitivity, improved spatial and temporal resolution, and the discriminatory power for measuring individual species of interest.

The editors would like to thank all of the authors for their excellent and timely contributions and the editorial staff of $\mathrm{ABC}$ for their invaluable assistance during the preparation of this special issue.

\section{References}

1. Wagner V, Dullaart A, Bock AK, Zweck A (2006) Nat Biotechnol 24:1211-1217

2. http://nihroadmap.nih.gov/nanomedicine/

3. http://www.nanopharmaceuticals.org/files/nanomedicine.pdf

4. Sanhai WR, Sakamoto JH, Canady R, Ferrari M (2008) Nature Nanotechnol 3:242-244

5. Carmo VA, Ferrari CS, Reis EC, Ramaldes GA, Pereira MA, De Oliveira MC, Cardoso VN (2008) Nucl Med Commun 29:33-38

6. Thompson M (2004) Analyst 129:671

7. Durner J (2010) Angew Chem Int Ed 49:1026-1051

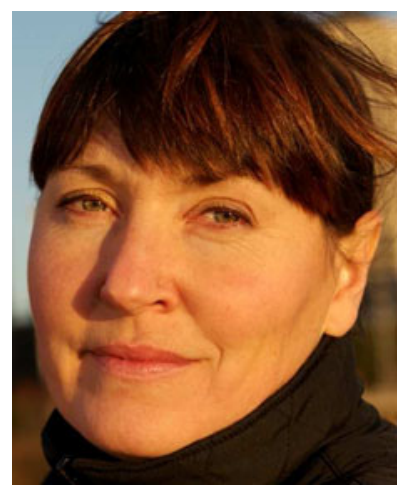

Christine Kranz is currently heading the surface analytical group at the Institute of Analytical and Bioanalytical Chemistry at the University of Ulm, Ulm, Germany. In addition, she has been a member of the Center for Cell and Molecular Signaling at Emory University, School of Physiology since 2005. She is working in the field of scanning probe microscopy, in particular, scanning electrochemical microscopy (SECM), multifunctional scanning probes (e.g., combination AFM-SECM, IR-

SECM, IR-AFM), and miniaturized amperometric biosensor technology, integrated microsystems, biomimetic sensors, and DNA sensing. Her research interests focus on the development of novel multifunctional scanning micro- and nanoprobe concepts, surface modification, and characterization with scanning probe techniques. In her function she is the scientific supervisor of the recently established FIB center UUlm. 


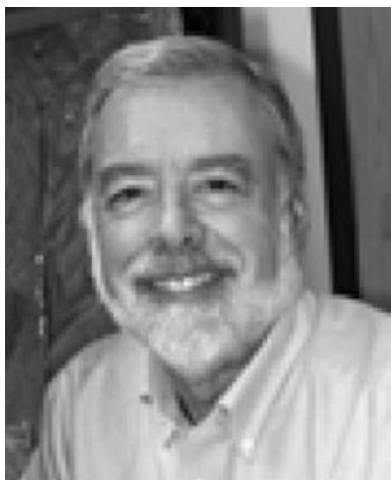

Douglas C. Eaton is a distinguished Professor and chair of Physiology at Emory University, Atlanta, GA, USA. The goal of his research is to examine the cellular signaling mechanisms, which regulate membrane ion transport and cellular homeostasis. To examine these signaling mechanisms, he applies contemporary methods of cellular and molecular biology including patch voltage clamp methods and expression of cloned signaling molecules in heterologous expression systems. There are three main areas of cellular signaling research in his laboratory: cellular responses involving steroid hormones and other lipid molecules, signaling mechanisms responsible for the responses of renal cells to growth factors and vasoactive substances like angiotensin II, and signaling mechanics that control fluid balance in the lungs.

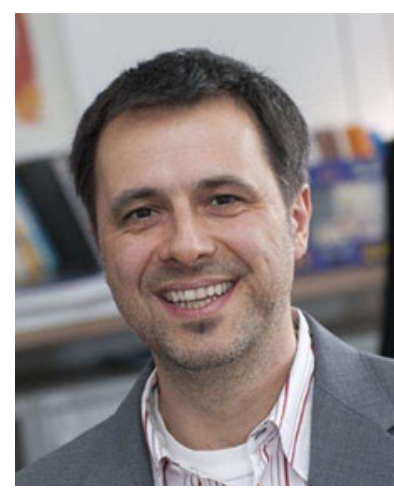

Boris Mizaikoff is currently Chaired Professor and Director of the Institute of Analytical and Bioanalytical Chemistry at the University of Ulm, Germany. $\mathrm{He}$ is also a member of the Center for Cell and Molecular Signaling at Emory University, Atlanta, GA. His research interests focus on optical sensors, biosensors, and biomimetic sensors operating in the mid-infrared spectral range, quantum cascade lasers, system miniaturization and integration based on micro- and nanofabrication, multifunctional scanning nanoprobes, scanning probe tip integrated nano(bio)sensors, focused ion beam techniques, development of chemical recognition layers for separation and sensing applications, chemometric data evaluation, environmental analytical chemistry, process analytical chemistry, and biomedical diagnostics. 